\author{
S. H. Rasouli and B. Salehi
}

\title{
ON THE EXISTENCE OF NONTRIVIAL SOLUTIONS FOR NONLOCAL ELLIPTIC KIRCHHOFF TYPE PROBLEMS WITH NONLINEAR BOUNDARY CONDITIONS
}

\begin{abstract}
In this paper, by using the Mountain Pass Lemma, we study the existence of nontrivial solutions for a nonlocal elliptic Kirchhoff type equation together with nonlinear boundary conditions.
\end{abstract}

KEY WORDs: Kirchhoff type problems, Mountain-Pass Lemma, nonlinear boundary conditions.

AMS Mathematics Subject Classification: 35J60, 35J20, 35J25.

\section{Introduction and preliminaries}

Consider the boundary value problem of Kirchhoff type

$$
\begin{cases}-\left(a+b \int_{\Omega}|\nabla u|^{2} d x\right) \Delta u=f(x, u), & x \in \Omega, \\ \frac{\partial u}{\partial n}=g(x, u), & x \in \partial \Omega,\end{cases}
$$

where $\Omega$ is a bounded domain with smooth boundary in $R^{N}$ for $N=1,2,3$, $a, b>0$, are real numbers, and $f, g$ are Carathéodory functions.

Recently, more researches were done about existence of nontrivial solutions to the classes of the Kirchhoff type problems by Mathematicians (See [3], [4], [5], [6], [7], [8]). Also in [2], Bitao Cheng studied the existence and multiplicity results of nontrivial solutions for nonlocal elliptic Kirchhoff type problem

$$
\begin{cases}-\left(a+b \int_{\Omega}|\nabla u|^{2} d x\right) \Delta u=f(x, u), & x \in \Omega, \\ u=0, & x \in \partial \Omega,\end{cases}
$$

under the following assumptions:

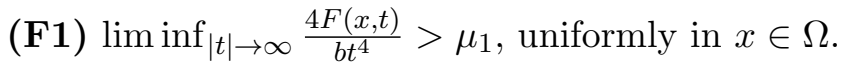


(F2) $\lim \sup _{t \rightarrow 0} \frac{2 F(x, t)}{a t^{2}}<\lambda_{1}$, uniformly in $x \in \Omega$.

(F3) $\liminf |t| \rightarrow \infty \frac{f(x, t) t-4 F(x, t)}{|t|^{\tau}}>-\alpha$, uniformly in $x \in \Omega$.

where $\lambda_{1}$ is the first of the eigenvalue problem

$$
\begin{cases}-\Delta u=\lambda u, & x \in \Omega \\ u=0, & x \in \partial \Omega,\end{cases}
$$

and $\mu_{1}$ is the first of the eigenvalue problem

$$
\begin{cases}-\left(\int_{\Omega}|\nabla u|^{2}\right) \triangle u=\mu u^{3}, & x \in \Omega, \\ u=0, & x \in \partial \Omega .\end{cases}
$$

Motivated by the results of the above works, we are interested in the existence of positive solutions for problem (1). Our main difficulty will be the nonlinearity of $g(x, u)$. To overcome this difficulty, we need to restrict the problem (1) to some assumptions.

Problem (1) is posed in the framework of the Sobolev space $X=H^{1}(\Omega)$ with the standard norm

$$
\|u\|^{2}=\int_{\Omega}|\nabla u|^{2} d x .
$$

Moreover, a function $u \in X$ is said to be a weak solution of problem (1) if

$$
\int_{\Omega} f(x, u) v d x=-\left(a+b\|u\|^{2}\right)\left[\int_{\Omega} v g(x, u) d x-\int_{\partial \Omega} \nabla u \nabla v d s\right],
$$

for all $v \in H$. It is well known that weak solutions of problem (1) correspond to critical points of the functional $I: X \rightarrow \mathbb{R}$

$$
I(u)=\frac{a}{2}\|u\|^{2}+\frac{b}{4}\|u\|^{4}-\int_{\Omega} F(x, u) d x-\int_{\partial \Omega} G(x, u) d x,
$$

where

$$
F(x, u)=\int_{0}^{u} f(x, t) d t, \quad G(x, u)=\int_{0}^{u} g(x, t) d t .
$$

The base of our work is finding critical points by using the mountain pass lemma which we describe below.

Definition 1. Let $X$ be a Banach space and $I \in C^{1}(X, R)$. We say that I satisfies the $(P S)$ condition if any sequence $\left\{u_{n}\right\} \subset X$ that $\left\{I\left(u_{n}\right)\right\}$ be bounded and $\left\{I^{\prime}\left(u_{n}\right)\right\} \longrightarrow \infty$ as $n \longrightarrow \infty$, possesses a convergent subsequence.

Lemma 1 (Mountain pass [1]). Let $X$ be a real Banach space and $I \in$ $C^{1}\left(X, R^{1}\right)$ satisfying $(P S)$ condition. Suppose 
(L1) there are constants $a, r>0$ such that for any $u \in X$ that $\|u\|=r$, we have

$$
I(u) \geq a>0
$$

(L2) there is $e \in X$ such that $I(e)<0$;

Then I possesses a critical value as

$$
C=\inf _{g \in \Gamma} \max _{t \in[0,1]} I(g(t)),
$$

where

$$
\Gamma=\{g \in C([0,1], X): g(0)=0, g(1)=e\} .
$$

Definition 2. We say that operator $J: X \longrightarrow X^{*}$ is satisfying in condition $(S)_{+}$, if $u_{n} \rightarrow u$ in $X$ and $\lim \sup _{n \rightarrow \infty}\left\langle J\left(u_{n}\right), x_{n}-x\right\rangle \leq 0$, implies $u_{n} \rightarrow u$ in $X$.

\section{Existence theorem}

We set

$$
\Upsilon(u)=\frac{a}{2}\|u\|^{2}+\frac{b}{4}\|u\|^{4} \Phi(u)=\int_{\Omega} F(x, u) d x, \quad \Psi(u)=\int_{\partial \Omega} G(x, u) d x
$$

where

$$
F(x, u)=\int_{0}^{u} f(x, t) d t, \quad G(x, u)=\int_{0}^{u} g(x, t) d t .
$$

Note that $\Upsilon^{\prime}: X \longrightarrow X^{*}$ such that $\left\langle\Upsilon^{\prime}(u), v\right\rangle=\left(a+b\|u\|^{2}\right) \int_{\Omega} \nabla u \nabla v d x$, is satisfying in conditions $(S)_{+}$, and is a homeomorphism.

Also we have $\Phi^{\prime}: X \longrightarrow X^{*}$ such that $\left\langle\Phi^{\prime}(u), v\right\rangle=\int_{\Omega} v g(x, u) d x, \forall v \in X$ and for any $v \in X$ we define $F: X^{*} \rightarrow \mathbb{R}$ such that $F(J)=J(v)$. Then $F \in X^{* *}$ and $F$ is linear and continuous. Since $F$ is finite rank then it is compact. Now if $\left\{u_{n}\right\}$ be bounded in $X$ then

$$
\begin{aligned}
\left\|\Phi^{\prime}\left(u_{n}\right)\right\| & =\sup \left\|\left\langle\Phi^{\prime}\left(u_{n}\right), v\right\rangle\right\|=\sup _{\|v\|=1}\left\|\int_{\Omega} f\left(x, u_{n}\right) v d x\right\| \\
& \leq \sup _{\|v\|=1} \int_{\Omega} f\left(x, u_{n}\right)\|v\| d x \leq \int_{\Omega}\|f\| d x=\|f\||\Omega|,
\end{aligned}
$$

since $\|f\|=\sup _{x \in \Omega, n \in N}\left|f\left(x, u_{n}\right)\right|$ and $\left\{u_{n}\right\} \subset \mathbb{R}$ is bounded and $f$ on $\mathbb{R}$ is continuous, then $\|f\|$ is finite, therefore $\left\|\Phi^{\prime}\left(u_{n}\right)\right\|<\infty$, i.e., $\left\{\Phi^{\prime}\left(u_{n}\right)\right\}$ is bounded. By the compactness of $F,\left\{F\left(\Phi^{\prime}\left(u_{n}\right)\right)\right\}$ possesses a convergent subsequence, then is satisfying in cauchy condition. It is easy to see that $\Phi^{\prime}$ is compact, so is continuous. Similarly $\Psi^{\prime}$ is compact and continuous. Then $I \in C^{1}(X, R)$. We now consider the following assumptions to state our main result: 
(H1) there exists $c_{1}>0$, such that $|f(x, t)| \leq c_{1} t^{p} ; 2<p<2^{*}$.

(H2) there exists $c_{2}>0$, such that $|g(x, t)| \leq c_{2} t^{q} ; 2<q<2^{*}$.

(H3) $\lim _{|t| \rightarrow \infty} \frac{F(x, t)}{|t|^{2}}=0$, uniformly for any $x$.

(H4) $\lim _{|t| \rightarrow \infty} \frac{G(x, t)}{|t|^{2}}=0$, uniformly for any $x$.

(H5) there exists $\Omega^{\prime} \subset \Omega$ such that $\left|\Omega^{\prime}\right|>0$, there exists $t_{0}>0$ such that for any $x \in \Omega^{\prime}$ we have $F\left(x, t_{0}\right)>0$.

Now we give our main result.

Theorem 1. Let (H1)-(H5) hold. Then the problem (1) has at least one nontrivial solution in $X$.

To prove Theorem 1, we require the following three lemmas:

Lemma 2. Under the conditions $G_{1}-G_{5}$, the functional defined $I$ in (5) is satisfying in $(P S)$ condition.

Proof. Let $\left\{u_{n}\right\} \subset X$ be such that $\left\{I\left(u_{n}\right)\right\}$ is bounded and $I^{\prime}\left(u_{n}\right) \longrightarrow 0$. We now show that, $\left\{u_{n}\right\}$ possesses convergent subsequence. As $\left\{I\left(u_{n}\right)\right\}$ is bounded, then there exists $K>0$ such that

$$
K \geq I\left(u_{n}\right)=\frac{a}{2}\|u\|^{2}+\frac{b}{4}\left\|u_{n}\right\|^{4}-\int_{\Omega} F\left(x, u_{n}\right) d x-\int_{\partial \Omega} G\left(x, u_{n}\right) d x .
$$

First we claim that $\left\{u_{n}\right\}$ is bounded. If $\left\{u_{n}\right\}$ be unbounded, then contains a subsequence as $\left\{u_{n_{j}}\right\}$, that $\left\|u_{n_{j}}\right\| \rightarrow \infty$ as $j \rightarrow \infty$. By the Poincar's inequality $\int_{\Omega}\left|\nabla u_{n_{j}}\right|^{2} \rightarrow \infty$. So for $j$ large enough we can consider $\left|\nabla u_{n_{j}}\right|^{2} \geq 1$. By $\left(G_{3}\right)$ and $\left(G_{4}\right)$ there exists $\left\{\varepsilon_{n}\right\}$ such that $\varepsilon_{n} \rightarrow 0$ as $n \rightarrow \infty$, and

$$
F\left(x, u_{n}\right) \leq \varepsilon_{n}\left|u_{n}\right|^{2}, \quad G\left(x, u_{n}\right) \leq \varepsilon_{n}\left|u_{n}\right|^{2} .
$$

Then

$$
\begin{aligned}
K \geq I\left(u_{n j}\right) & =\frac{a}{2}\left\|u_{n_{j}}\right\|^{2}-\varepsilon_{n} \int_{\Omega}\left|u_{n_{j}}\right|^{2} d x-\varepsilon_{n} \int_{\partial \Omega}\left|u_{n_{j}}\right|^{2} d x \\
& =\frac{a}{2}\left\|u_{n_{j}}\right\|^{2}-\varepsilon_{n}\left(\int_{\Omega}\left|u_{n_{j}}\right|^{2} d x+\int_{\partial \Omega}\left|u_{n_{j}}\right|^{2} d x\right) .
\end{aligned}
$$

On the other hand, by the Poincar's inequality there exists $c_{0}>0$, such that

$$
\int_{\Omega}\left|u_{n_{j}}\right|^{2} d x \leq c_{0} \int_{\Omega}\left|\nabla u_{n_{j}}\right|^{2} d x
$$

Consequently,

$$
K \geq I\left(u_{n_{j}}\right)=\frac{a}{2}\left\|u_{n_{j}}\right\|^{2}-\varepsilon_{n} c_{0}\left(\int_{\Omega}\left|\nabla u_{n_{j}}\right|^{2} d x+\frac{1}{c_{0}} \int_{\partial \Omega}\left|u_{n_{j}}\right|^{2} d x\right) .
$$


If we take

$$
\varepsilon_{n}=\frac{1}{\int_{\Omega}\left|\nabla u_{n_{j}}\right|^{2} d x+\frac{1}{c_{0}} \int_{\partial \Omega}\left|u_{n_{j}}\right|^{2} d x},
$$

then $K \geq \frac{a}{2}\left\|u_{n_{j}}\right\|^{2}-c_{0}$. This is contradiction. Therefore, $\left\{u_{n}\right\}$ is bounded in $X$. So, by the reflexivity of $X,\left\{u_{n}\right\}$ has a week convergent subsequence in $X$ like $\left\{u_{n_{k}}\right\}$. Hence, due to the compactness $\Phi^{\prime}$ and $\Psi^{\prime}$ we have that

$$
\Phi^{\prime}+\Psi^{\prime} \rightarrow u .
$$

Since $I=\Upsilon-\Phi-\Psi$ and $I^{\prime}\left(u_{n_{k}}\right) \longrightarrow 0$ then

$$
\Upsilon^{\prime}\left(u_{n_{k}}\right) \longrightarrow \Phi^{\prime}\left(u_{n_{k}}\right)+\Psi^{\prime}\left(u_{n_{k}}\right)
$$

Since $\Upsilon^{\prime}$ is homeomorphism, we can conclude that

$$
u_{n_{k}} \longrightarrow\left(\Upsilon^{\prime}\right)^{-1}(u)
$$

Lemma 3. There exists $r>0$ such that for every $u \in X$, with $\|u\|=r$ we have $I(u)>0$.

Proof. By $\left(G_{1}\right)$ and $\left(G_{2}\right)$ for every $u \neq 0, t \in R$ we see that

$$
\begin{aligned}
I(t u) & =\frac{a}{2} t^{2}\|u\|^{2}+\frac{b}{4} t^{4}\|u\|^{4}-\int_{\Omega} F(x, t u) d x-\int_{\partial \Omega} G(x, t u) d x \\
& \geq \frac{a}{2} t^{2}\|u\|^{2}-\max \left\{c_{1}, c_{2}\right\}|t|^{\max \{p, q\}}\left[\int_{\Omega}|u|^{2} d x+\int_{\partial \Omega}|u|^{2} d x\right] \\
& =t^{2}\left[\frac{a}{2}\|u\|^{2}-\max \left\{c_{1}, c_{2}\right\}|t|^{\max \{p, q\}-2}\left(\int_{\Omega}|u|^{2} d x+\int_{\partial \Omega}|u|^{2} d x\right)\right] .
\end{aligned}
$$

Therefore, for every $t$ small enough $I(t u)>0$. Now for any $t$ that $I(t u)>0$ we can take $r=\|t u\|$.

Lemma 4. There exists $e \in X$ such that $I(e)<0$.

Proof. Define

$$
u(x)= \begin{cases}1, & x \in \Omega, \\ 0, & x \in \Omega-\Omega^{\prime} .\end{cases}
$$

Then $\int_{\Omega}|\nabla u|^{2} d x=0$. By $\left(G_{5}\right)$ we have

$$
I\left(t_{0} u\right)=-\int_{\Omega} F\left(x, t_{0} u\right) d x-\int_{\partial \Omega} G\left(x, t_{0} u\right) d x .
$$


Since $G\left(x, t_{0} u\right)=G(x, 0)=0$, for all $x \in \partial \Omega$, and

$$
\begin{aligned}
\int_{\Omega} F\left(x, t_{0} u\right) d x & =\int_{\Omega^{\prime}} F\left(x, t_{0}\right) d x-\int_{\Omega-\Omega^{\prime}} F(x, 0) d x \\
& =\int_{\Omega^{\prime}} F\left(x, t_{0}\right)>0,
\end{aligned}
$$

we conclude that $I\left(t_{0} u\right)<0$. Therefore by choosing $e=t_{0} u$ the lemma is proved.

Now, we complete the proof of Theorem 1. By Lemmas 2-4, the conditions of Mountain Pass Lemma are satisfied. Therefore, $I$ has a nontrivial critical point as

$$
C=\inf _{g \in \Gamma} \max _{t \in[0,1]} I(g(t))
$$

that

$$
\Gamma=\left\{g \in C([0,1], X) ; g(0)=0, g(1)=t_{0} u\right\} .
$$

Then the problem (1) has a nontrivial solution and also Lemma 3 implies that $C$ is positive.

\section{References}

[1] Ambrosetti A., Rabinowitz P.H., Dual varitional methods in critical point theory and applications, J. Funct. Anal., 14(1973) 349-381.

[2] Cheng B., New existense and multiplicity of nontrivial solutions for nonlocal elliptic Kirchhoff type problems, J. Math. Anal. Appls, (2012).

[3] Cheng B., Wu X., Existence results of positive solution of Kircchoff problems, Nonl. Anal., 71 (2009) 4883-4892.

[4] Cheng B., Wu X., Multiplicity of nontrivial solutions for Kirchhoff type problems, Hindawi Publishing Corporation, Boundary Value Problems Volume 2010. Article ID 268946, 13 page, DOI:10.1155/2010/268946.

[5] Alves C.O., Correa F.J.S.A., Ma T.F., Positive solutions for a quasilinear elliptic equation of Kirchhoff type, Comput. Math. Appl., 49(2005), 85-93.

[6] He X., Zou W., Infinitely many positive solutions for Kirchhoff-type problems, Nonl. Anal., 70(2009), 1407-1414.

[7] Ma T.F., Munoz Rivera J.E., Positive solutions for a nonlinear nonlocal elliptic transmisson problem, Apple. Math. Lett., 16(2003), 243-248.

[8] Perea K., Zhang Z., Nontrival solutions of Kirchhoff-type problems via the Yang index, J. Diff. Eqs., 221(1)(2006), 246-255.

$$
\begin{aligned}
& \text { S. H. Rasouli and B. Salehi } \\
& \text { Department of Mathematics } \\
& \text { FACUlty of Basic ScIENCES } \\
& \text { Babol University of TeChNology } \\
& \text { BABOL, IRAN } \\
& \text { e-mail: s.h.rasouli@nit.ac.ir }
\end{aligned}
$$

Received on 17.09.2014 and, in revised form, on 04.11.2015. 